Ortega-Arroyo, E. J., Jiménez-Pérez, J., Villanueva-Díaz, J., Yerena-Yamallel, J. I., Alanís-Rodríguez, E., \& AguirreCalderón, O. A. (2021). Potencial dendroclimático del encino rojo, Quercus sideroxyla (Fagaceae) en México. Revista de Biología Tropical, 69(3), 888-897. https://doi. org/10.15517/rbt.v69i3.44082

Biología Tropical

https://doi.org/10.15517/rbt.v69i3.44082

\title{
Potencial dendroclimático del encino rojo, Quercus sideroxyla (Fagaceae) en México
}

Edgar Jesús Ortega-Arroyo ${ }^{1 *}$; (D) https://orcid.org/0000-0002-6703-5959

Javier Jiménez-Pérez ${ }^{1}$; (D) https://orcid.org/0000-0003-4684-1086

José Villanueva-Díaz;; (D https://orcid.org/0000-0001-8211-1203

José Israel Yerena-Yamallel'; (D) https://orcid.org/0000-0002-9216-7427

Eduardo Alanís-Rodríguez; ; (D) https://orcid.org/0000-0001-6294-4275

Oscar Alberto Aguirre-Calderón1; (D) https://orcid.org/0000-0001-5668-8869

1. Facultad de Ciencias Forestales, Universidad Autónoma de Nuevo León, km 145, carretera nacional 85, C.P.67700, Linares, Nuevo León, México; ortega.uan1@gmail.com (*Correspondencia),jjimenez20@gmail.com, israel.yerena@gmail.com, eduardoforestal@gmail.com,oaguirre16@gmail.com

2. Laboratorio de Dendrocronología, INIFAP CENID-RASPA, km 6.5 Margen derecha del canal de sacramento, C.P. 35140, Gómez Palacio, Durango, México; villanueva.jose@inifap.gob.mx

Recibido 02-X-2020. Corregido 02-VII-2021. Aceptado 24-VIII-2021.

\section{ABSTRACT \\ Dendroclimatic potential of red oak, Quercus sideroxyla (Fagaceae) in Mexico}

Introduction: Dendrochronological studies in Mexico have been mainly based on conifers, while hardwood species have been little studied. This has been the case of the genus Quercus, which has a high taxonomic diversity in the country but has not been previously studied for dendrochronological issues, despite the ecological and economic values of oak species.

Objective: In the present investigation, the dendroclimatic potential of Quercus sideroxyla in Northwestern Mexico was determined, as well as its relationship with climatic variables such as precipitation and temperature. Methods: The research was carried out in the state of Durango, Chavarría Viejo with coordinates (234' $\mathrm{N} \&$ $\left.-105^{\circ} 33^{\prime} \mathrm{W}\right)$. Samples of $5 \mathrm{~cm}$ to $7 \mathrm{~cm}$ were collected in two sites under forest exploitation and processed by conventional dendrochronological techniques.

Results: A chronology of total ring width was developed, which was composed of 30 samples from 16 trees for the period from 1917 to 2018 (101 years). Inter-series intercorrelation values of 0.43 , mean sensitivity of 0.36 , signal-to-noise ratio of 3.53 and first-order autocorrelation (0.58) were obtained. Regarding the climate-growth relationship, the ring width index values were correlated with data from the climatic station nearest to the study site, where winter-spring precipitation (January-May) was the variable with the greatest influence on the growth of the species.

Conclusions: Based on the dendrochronological parameters, the high potential of the species that were used in dendroclimatic studies in the region has demonstrated that the response of the species to precipitation is similar to that of the conifers with which the Quercus sideroxyla shares its habitat with.

Key words: growth rings; anatomical characteristics; dendrochronology; climatic influence; oak. 
Para comprender cómo fue el comportamiento de la variabilidad climática en el pasado, se han empleado diferentes tipos de fuentes sustitutas conocidas como registros indirectos (proxy en inglés). La dendrocronología, mediante el estudio de los anillos de crecimiento fechados al año exacto de su formación, una de las de mayor resolución, permite realizar la reconstrucción del clima pasado a relación interanual y multianual (Fritts, 1976). En México, diversos géneros han sido empleados en estudios sobre potencial dendrocronológico y dendroclimático, entre los que sobresalen especies de coníferas de Pinus, Pseudotsuga y Taxodium (Acosta-Hernández et al., 2017). Sin embargo, en la búsqueda de otros géneros como el Quercus, el panorama se amplía, ya que de las 500 especies que existen en todo el mundo, alrededor de 130 a 150, se encuentran en México (Luna-José et al., 2003; Romero Rangel et al., 2002), donde su potencial dendrocronológico y respuesta climática aún no han sido del todo estudiados. Los estudios sobre crecimiento radial en especies pertenecientes al género Quercus para México, se han enfocado principalmente en la descripción anatómica (De $\mathrm{La}$ Paz Pérez-Olvera \& Dávalos-Sotelo, 2008) y algunos han tratado sobre poder calorífico y densidad de la madera (Herrera-Fernández et al., 2017). En referencia a su respuesta al clima, estudios sobre el potencial hídrico y osmótico de los encinos (especies del género Quercus) en el noreste de México, revelan la capacidad para superar periodos de sequía, logrando adaptarse ante cambios drásticos en ambientes dinámicos (Himmelsbach, 2009). Por lo antes descrito, los objetivos de esta investigación son: a) aplicar técnicas dendrocronológicas y datar el año exacto de formación de la serie de crecimiento del encino (anillo total), b) analizar el potencial dendrocronológico del $Q$. sideroxyla y c) analizar la influencia de las variables climáticas como temperatura y precipitación en el ancho de anillo total de $Q$. sideroxyla.

\section{MATERIALES Y MÉTODOS}

Área de estudio: La presente investigación se desarrolló en el estado de Durango, en los parajes: Mesa de Alferes y Mesa de la Mula, en el ejido Chavarría Viejo perteneciente al municipio de Pueblo Nuevo, Durango ubicado a 3.5 kilómetros del camino El Salto-Las Adjuntas $\left(23^{\circ} 43^{\prime} \mathrm{N} \& 105^{\circ} 33^{\prime} \mathrm{W}\right)$ a una altitud de 2500 m.s.n.m. (Valenzuela Nuñez \& Granados Sánchez, 2009). El tipo de clima es templado, subhúmedo, con una temperatura media anual de $12{ }^{\circ} \mathrm{C}$ y una precipitación media anual de $1200 \mathrm{~mm}$. La temperatura del mes más frío oscila entre -3 y $18{ }^{\circ} \mathrm{C}$ y la temperatura del mes más cálido es de $22{ }^{\circ} \mathrm{C}$ y la precipitación en el mes más seco es menor a $40 \mathrm{~mm}$ (García, 2004). El área se encuentra bajo aprovechamiento forestal, y la vegetación dominante es bosque de pino encino, siendo Pinus durangensis Martínez, P. cooperi C.E. Blanco y $P$. arizonica Engelm. las que presentan mayor dominancia, seguidas de Quercus sideroxyla y Q. rugosa Née (Cruz-Cobos et al., 2008).

Tipo de muestreo y obtención de muestras: El muestreo se realizó de manera selectiva en árboles $\geq 20 \mathrm{~cm}$ de diámetro a la altura del pecho (DAP). El criterio de selección consistió en que los especímenes tuvieran de preferencia un fuste recto y que se encontraran bajo aprovechamiento, de acuerdo con Villanueva-Díaz et al. (2009a) de cada árbol seleccionado se tomaron dos rodajas (secciones transversales) de la base del árbol, con un grosor de 5-7 cm.

Preparación de las muestras: Las muestras se procesaron mediante las técnicas dendrocronológicas convencionales (Stokes \& Smiley, 1968) en el Laboratorio de Dendrocronología del Centro Nacional de Investigación Disciplinaria Relación Agua, Suelo, Planta, Atmósfera, Instituto Nacional de Investigaciones Forestales Agrícolas y Pecuarias 
(CENID-RASPA, INIFAP). Las muestras se secaron a temperatura ambiente y posteriormente fueron pulidas con lijas de diferente grosor para facilitar la observación de los anillos de crecimiento (Fig. 1) a través de un estereomicroscopio Leica EZ4D de alta resolución ( $8 \mathrm{X}$ hasta $35 \mathrm{X}$ ). Durante el proceso de prefechado de las secciones se tomaron en consideración las recomendaciones sugeridas por otros autores, por ejemplo, sobre como limpiar repetidas veces las muestras con aire a presión o con un pincel, con el objetivo de evitar que los poros se tapen por efecto del pulido y así definir los límites del anillo anual con mayor facilidad (De La Paz Pérez-Olvera \& DávalosSotelo, 2008).

\section{Identificación del patrón de crecimien-} to: La datación visual o prefechado es uno de los principios más importantes de la dendrocronología (Constante-García et al., 2009) el cual consiste en la comparación de los crecimientos de las muestras procedentes de una misma área (Constante-García, 2015), identificando un patrón de crecimiento común. La datación, se logra al analizar y sincronizar una gran cantidad de series de crecimiento de distintos árboles para un sitio específico (Stokes \& Smiley, 1968). Este procedimiento permite detectar anillos falsos y ausentes. Su aplicación conlleva a tener un control experimental, que determina el año en que fue desarrollado cada anillo (Fritts, 1976; Swetnam et al., 1985). Una vez definido dicho patrón se procedió a realizar un pre-datado de los crecimientos arbóreos, definiendo el año, donde se desarrolló cada crecimiento anual.

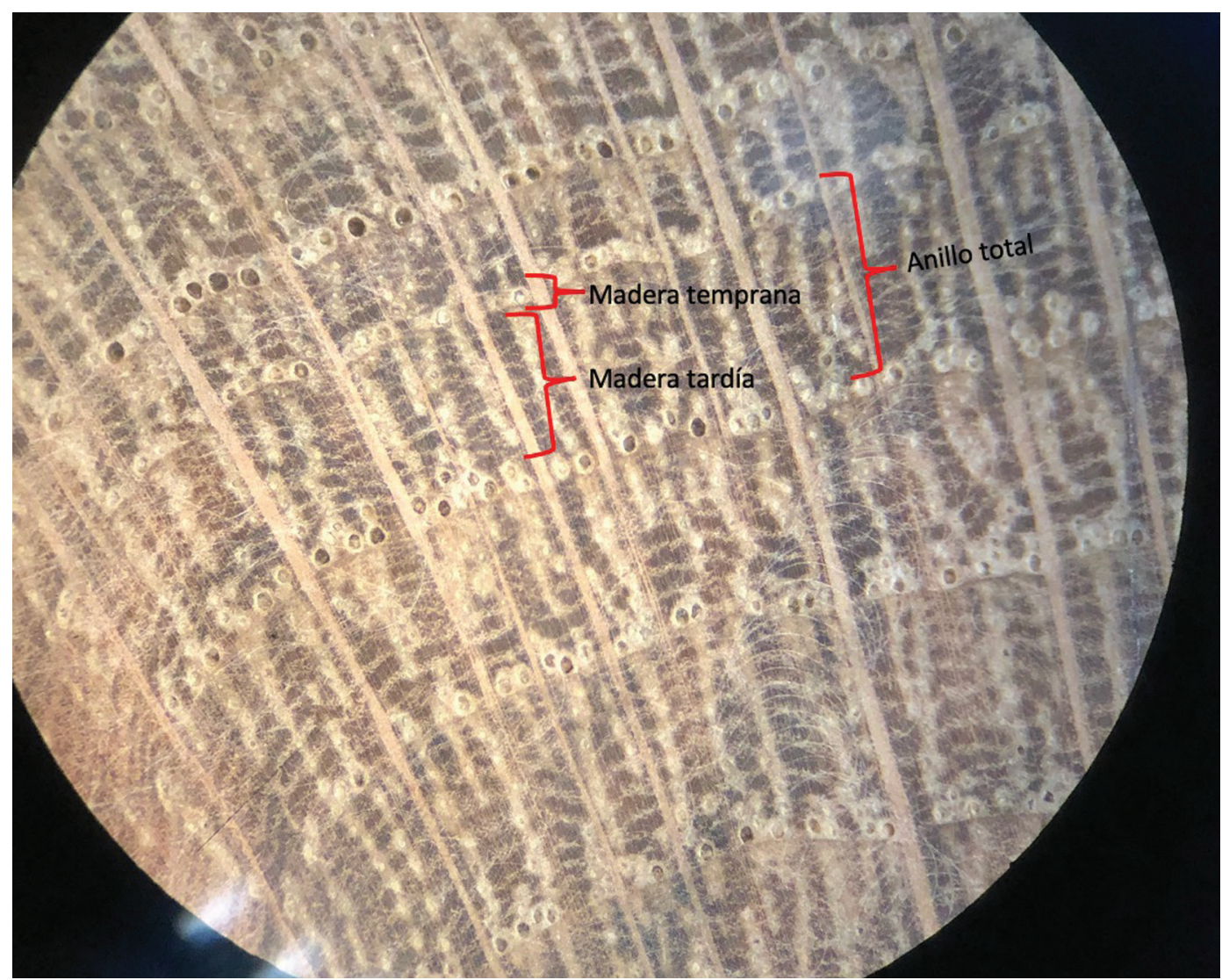

Fig. 1. Definición de los límites de madera temprana, tardía y anillo total en Quercus sideroxyla.

Fig. 1. Definition of earlywood, latewood, and total ring limits in Quercus sideroxyla. 
La medición de los anillos datados se realizó con el equipo de medición VELMEX con una precisión $0.001 \mathrm{~mm}$, y con el software MeasureJ2X para Windows (VoorTech, 2014). Los datos de la medición de los anillos de crecimiento se analizaron con el programa COFECHA, incluido en la Librería de Programas Dendrocronológicos de la Universidad de Arizona (DPL por sus siglas en inglés) (Grissino-Mayer, 2001; Holmes, 1983), cuya función es verificar la calidad del fechado o datación (Holmes, 1983).

La estandarización, proceso mediante el cual, a los valores contenidos en una serie de datos, se les extrae el valor de la media y al valor residual resultante de este proceso, se divide entre la desviación estándar de la muestra (Villanueva-Díaz et al., 2010) se efectuó con el programa ARSTAN. Este programa ajusta a cada serie, una curva exponencial negativa o alguna otra de mejor ajuste, con el objetivo de eliminar la varianza debido a factores biológicos (Cook \& Holmes, 1986; Delgado, 2000). Para este estudio, se utilizó la cronología estándar, que preserva la autocorrelación temporal.

Parámetros para definir el potencial dendrocronológico: De manera general, una especie se considera con buen potencial dendrocronológico, cuando ésta tiene la capacidad para desarrollar anillos de crecimiento de forma anual, reflejando así la variabilidad climática a través de la variación en el grosor de sus anillos de crecimiento anual (Fritts, 1976). Los estudios enfocados a evaluar el potencial dendrocronológico se fundamentan principalmente en cuatro criterios: a) sensibilidad media, b) desviación estándar, c) autocorrelación de primer orden y d) proporción señal-ruido (Bartens et al., 2012; Speer, 2010), además de considerar la señal expresada de la población (EPS, por sus siglas en inglés del "Expressed Population Signal"), parámetro que proporciona una estimación de la representatividad de una cronología con número limitado de muestras en comparación a la misma, si se considerara un número infinito de muestras (Wigley et al., 1984).
Relación clima-crecimiento: Para definir el período de precipitación, así como de temperatura que influye en el crecimiento de la especie, se utilizaron registros de las estaciones meteorológicas cercanas al sitio de muestreo, seleccionadas del Sistema Meteorológico Nacional de la Comisión Nacional del Agua (Servicio Meteorológico Nacional-Comisión Nacional del Agua, 2019). La estación más cercana al sitio de muestreo fue El Salto 10093, y cumple con el $90 \%$ de datos instrumentales fiables. La información considerada fue la precipitación pluvial mensual y temperatura mínima y máxima mensual del período 19632016. Con la selección de dicha información, se realizó un análisis de correlación de Pearson con la finalidad de detectar los meses o periodos estacionales donde surgían las correlaciones significativas $(\mathrm{P}<0.05)$ entre las series de índices de crecimiento (serie estándar) y los datos climáticos instrumentales de temperatura y precipitación.

\section{RESULTADOS}

Variables dendrocronológicas: En el muestreo se recolectaron 32 secciones transversales procedentes de 16 encinos de las cuales, se utilizaron sólo aquellas que presentaron mejor intercorrelación entre series (30 muestras, $94 \%$ del material recolectado). La serie dendrocronológica de anillo total se extendió de 1917 a 2018 (101 años de longitud) y, acorde con el número de muestras que intervinieron en el cálculo del valor del índice de cada año, el período de mayor confiabilidad es a partir del año 1950 en donde se presentan más de 7 muestras (Fig. 2) y se alcanza el umbral del 0.85 para el EPS.

Criterios dendrocronológicos de Quercus sideroxyla: En la Tabla 1 se muestran los valores resultantes a fin de determinar su potencial en estudios dendrocronológicos, los valores de cada variable, se ubican dentro del intervalo reportado por otros autores (Villanueva-Díaz et al., 2004). 


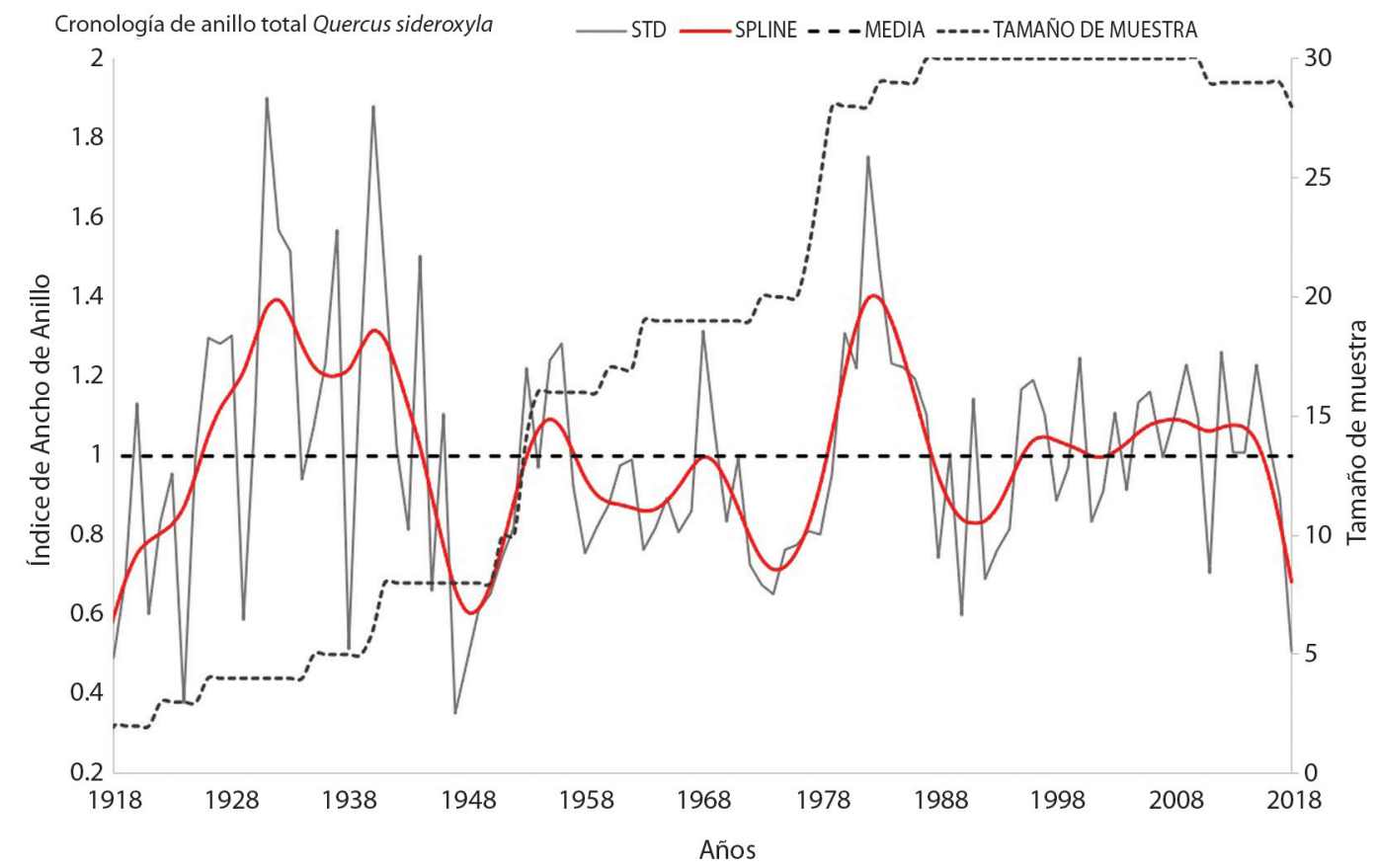

Fig. 2. Serie dendrocronológica estándar de anillo total y tamaño de muestra empleado para generar la cronología con extensión de 1917-2018 (101 años).

Fig. 2. Standard total ring dendrochronological series and sample size used to generate the chronology with extension 19172018 (101 years).

TABLA 1

Criterios específicos para determinar el potencial dendrocronológico de Quercus sideroxyla

TABLE 1

Specific criteria to determine the dendrochronological potential of Quercus sideroxyla

\begin{tabular}{lcc}
\hline \multicolumn{1}{c}{ Cuercus sideroxyla } \\
\hline Númerios de series (\# árboles) & Valores obtenidos en esta investigación & Rangos (Villanueva et al., 2004) \\
Intercorrelación entre series & $30(16)$ & $0.4-0.9$ \\
Desviación estándar & 0.434 & Varía con especie y sitio \\
Sensibilidad media & 1.009 & $0.15-0.68$ \\
Autocorrelación de 1er orden & 0.359 & $0.3-0.8$ \\
Relación señal-ruido & 0.58 & $1.0-20.0$ \\
Periodo & 3.531 & \\
\hline
\end{tabular}

Relaciones crecimiento-clima de Quercus sideroxyla: La base de datos procedentes de la estación climática mostró una extensión de 53 años (1963-2016), en donde la temperatura mínima presente en octubre influye de manera positiva en el desarrollo del encino $(\mathrm{R}=0.21$, $\mathrm{P}<0.05$ ), mientras que la temperatura máxima fue significativa para octubre y diciembre $(\mathrm{R}=$ $0.28, \mathrm{P}<0.05$ ), en lo que respecta a la precipitación de mayo que tuvo la mayor correlación $(\mathrm{R}=0.50, \mathrm{P}<0.05)$ con el índice de ancho de anillo (Fig. 3).

Sin embargo, al realizar el acumulado de los meses de enero a mayo se determinó que 


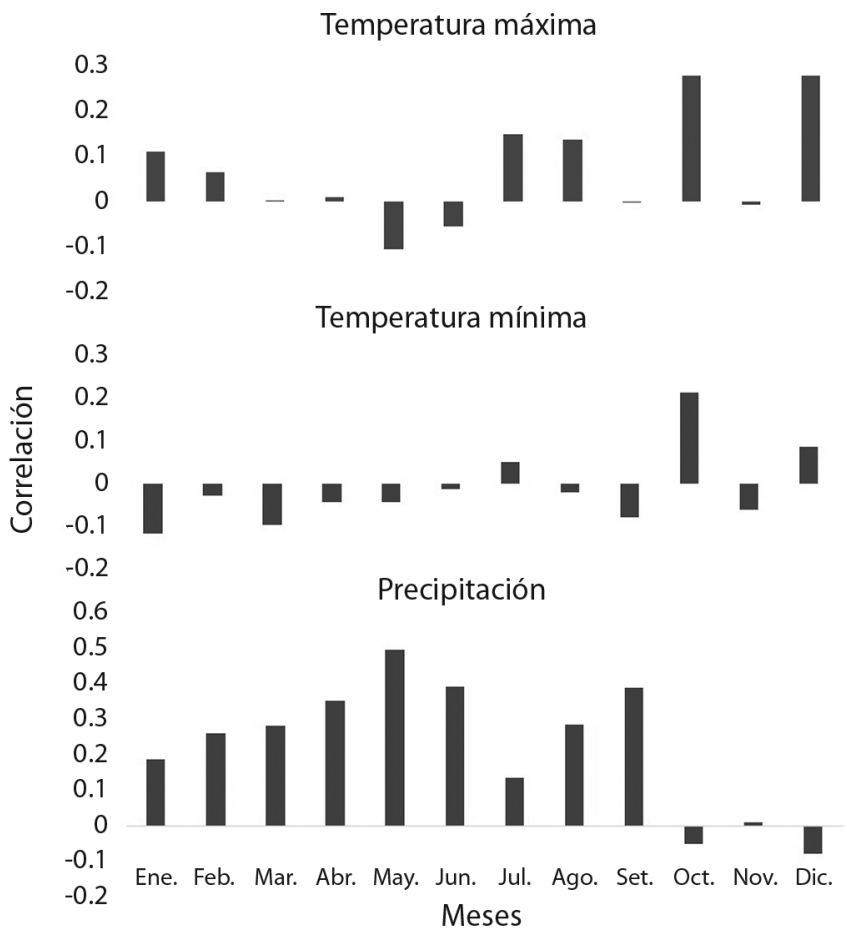

Fig. 3. Correlación entre la temperatura mínima, máxima y precipitación con el índice estandarizado de ancho de anillo. Fig. 3. Correlation between minimum temperature, maximum temperature, and precipitation with the standardized ring width index.

entre los registros de la precipitación pluvial presente en esos meses y el índice de ancho de anillo en su versión estándar de Quercus sideroxyla la correlación incrementó $(\mathrm{R}=0.51, \mathrm{P}<$ 0.05) (Fig. 4).

La precipitación acumulada de los meses (enero a mayo) mostró una correlación positiva $(\mathrm{R}=0.57, \mathrm{P}<0.05)$ con la serie de ancho de anillo total (Fig. 5), indicativo de que la especie explica el $50 \%$ de la variabilidad en la precipitación en el periodo estacional invierno-primavera).

\section{DISCUSIÓN}

El programa COFECHA (Holmes, 1983) establece como intercorrelación entre series mínima, un valor de $0.33(\mathrm{P}<0.01)$, para que se considere una correlación aceptable. Los valores estadísticos en esta investigación se encuentran dentro de los rangos reportados por Constante-García (2015), en un estudio reciente en el noreste de México con Quercus canbyi Trel. donde obtuvo una intercorrelación entre series de 0.70 , con una sensibilidad media de 0.40. Sin embargo son similares a los estimados por Díaz-Ramírez et al. (2016) para Pinus durangensis donde se obtuvieron valores de intercorrelación entre series de 0.46 y una sensibilidad media de 0.31 , aunque por debajo a los calculados por (Pompa-García et al., 2014) en Pinus cooperi, donde el valor de intercorrelación entre series fue de 0.61 y la sensibilidad media de 0.45 , sin embargo los valores reportados en esta investigación son consistentes con los obtenidos por Ortiz-Quijano et al. (2018) donde obtuvieron una intercorrelación entre series de 0.43 y una sensibilidad media de 0.18 con Fagus grandifolia subsp. mexicana en el estado de Hidalgo. Lo antes señalado refiere que el encino rojo tiene un potencial dendrocronológico para ser considerado en estudios de tipo dendroclimáticos en la región, debido a que la especie capta una variación significativa 


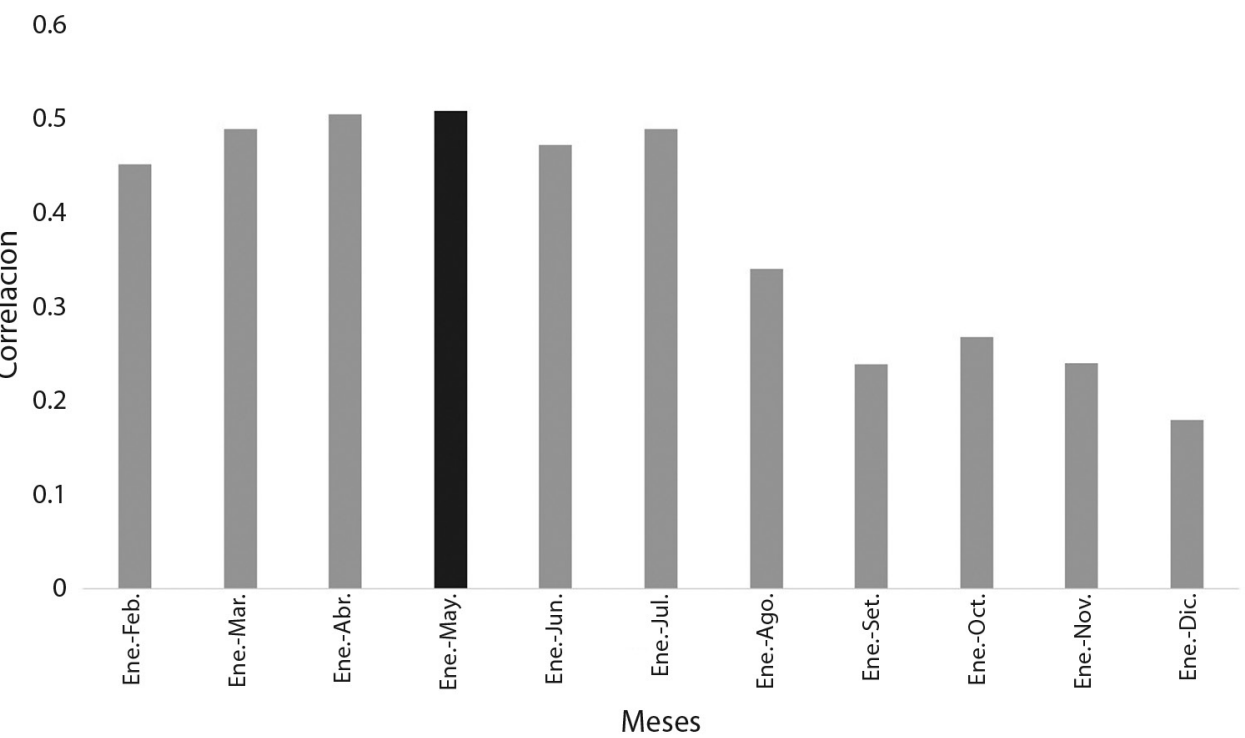

Fig. 4. Máxima correlación para el periodo de respuesta de la especie a la precipitación acumulada de enero a mayo (barra de color negro) del período de 1963-2016.

Fig. 4. Maximum correlation for period of species response to accumulated precipitation from January to May (black bar) for the period 1963-2016.

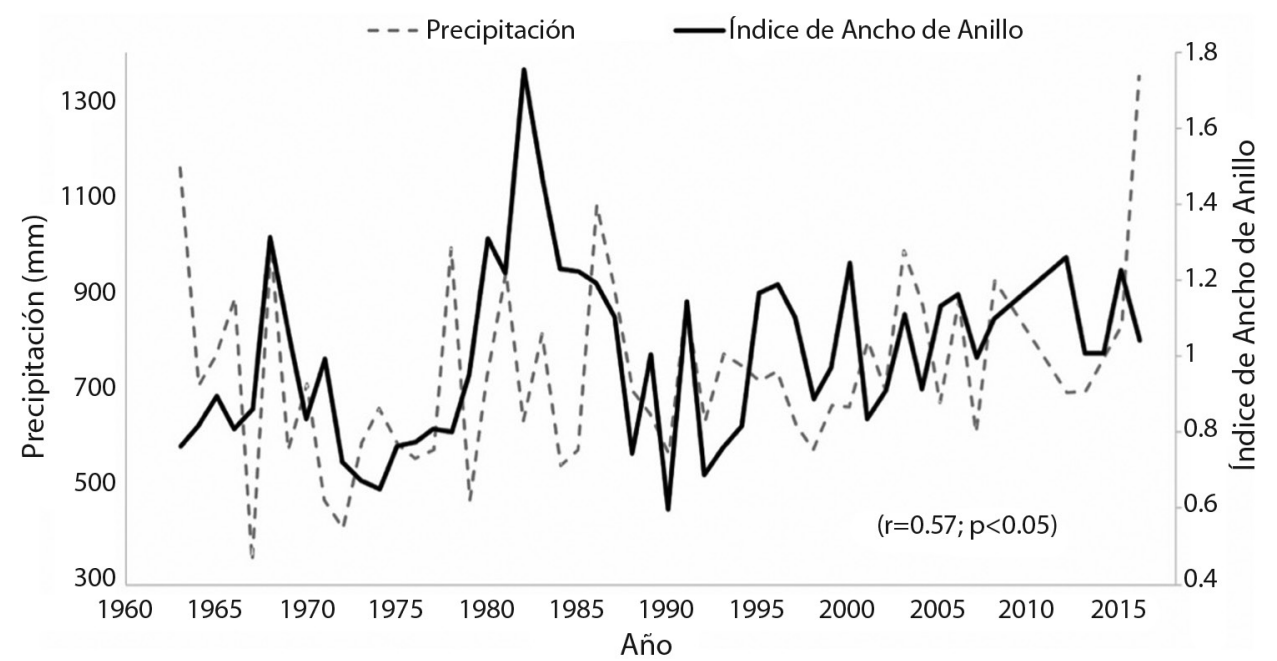

Fig. 5. Asociación entre la precipitación mensual acumulada y el índice estandarizado de ancho de anillo. La línea solida en color negro, se refiere al índice de ancho de anillo y la línea en color gris punteada, a la precipitación acumulada de enero a mayo.

Fig. 5. Association between monthly accumulated precipitation and the standardized ring width index. The solid black line refers to the ring width index and the dotted gray line to the accumulated precipitation from January to May.

de las condiciones climáticas dominantes en el área de estudio, que es una de las premisas para considerar una especie con potencial dendroclimático (Stahle, 1999).
Con respecto a los valores de autocorrelación de primer orden, a pesar de que el valor obtenido de 0.58 . A pesar de ser alto se considera como adecuado, de acuerdo con el 
intervalo de valores que menciona VillanuevaDíaz et al. (2004), mencionan rangos que oscilan entre 0.3 a 0.8 .

En los resultados de la relación señal-ruido, el valor obtenido (3.53) puede considerarse como bajo, de acuerdo con lo reportado por Díaz-Rámirez et al. (2016). Este valor resultante se debe a que el área se encuentra bajo aprovechamiento forestal $\mathrm{y}$, aunque por lo general los valores superiores a 5 y 6 se consideran adecuados, los valores obtenidos en esta investigación se consideran aceptables, con fines dendrocronológicos (Wigley et al., 1984).

Las coníferas del norte de México responden a la precipitación que se presenta en la estación invierno-primavera (Villanueva-Díaz et al., 2014), debido a la baja intensidad de las lluvias y de la alta capacidad de infiltración de los suelos, lo cual favorece que gran parte se quede almacenada en el suelo y sea aprovechada al inicio de la estación de crecimiento.

Para $Q$. sideroxyla, la respuesta a la variable precipitación fue similar a la de las coníferas de acuerdo a lo reportado por Villanueva Díaz et al. (2009b) y por Chávez-Gándara et al. (2017) en donde demostraron que la precipitación presente en el período invierno primavera, es la que mayormente impacta en el crecimiento de especies de coníferas, lo cual se explica a su vez por los tipos de bosque de pino-encino en donde se desarrollan, así mismo Ortiz-Quijano et al. (2018), en un estudio desarrollado más al centro de México, obtuvieron valores significativos con la precipitación $(\mathrm{R}=0.66, \mathrm{P}<0.01)$ presente de enero a junio, logrando identificar periodos de sequía y periodos de abundante precipitación en la cronología desarrollada para Fagus grandifolia subsp. mexicana.

En cuanto a la correlación con la temperatura mínima de octubre, distintos autores mencionan la capacidad de los especies forestales para aprovechar estas temperaturas (Cerano Paredes et al., 2012), a los valores obtenidos en esta investigación se le puede atribuir a que en ese mes se presenta una menor evaporación y por lo tanto existe una mayor humedad en el suelo, por otro lado, la temperatura máxima también benefició el desarrollo de la especie principalmente en octubre y diciembre, esto se puede explicar debido a que a temperaturas elevadas incrementan el déficit de evapotranspiración el cual es aprovechado por esta especie para su crecimiento

Los valores de los criterios dendrocronológicos obtenidos del ancho de anillo total de Quercus sideroxyla muestran que esta especie puede ser utilizada para evaluar relaciones entre clima y crecimiento radial debido a que sus parámetros dendrocronológicos se ubican dentro de los rangos analizados por otros autores.

La cronología de ancho de anillo total se desarrolló para el período comprendido entre 1917 y 2018 (101 años) y respondió de forma positiva a la precipitación acumulada desde enero hasta mayo.

Con base en los criterios dendrocronológicos específicos, se demuestra el potencial de $Q$. sideroxyla para ser empleado en estudios dendroclimáticos futuros en la región y donde se distribuya, así mismo se constituye como la primera especie para el inicio de una red dendrocronológica.

Declaración de ética: los autores declaran que todos están de acuerdo con esta publicación y que han hecho aportes que justifican su autoría; que no hay conflicto de interés de ningún tipo; y que han cumplido con todos los requisitos y procedimientos éticos y legales pertinentes. Todas las fuentes de financiamiento se detallan plena y claramente en la sección de agradecimientos. El respectivo documento legal firmado se encuentra en los archivos de la revista.

\section{AGRADECIMIENTOS}

Los autores agradecen al CONACYT por la beca otorgada al primer autor para realizar los estudios de Doctorado en Ciencias con especialidad en Manejo de Recursos Naturales 001415 (No. Becario 705248), así mismo se agradece al Ejido Chavarría Viejo, en especial a Miguel Ángel Alvarado Huízar por las facilidades otorgadas para la toma de las muestras en 
campo, y al laboratorio de Dendrocronología del INIFAP CENID-RASPA para procesar el material recolectado, así como a los comentarios de dos revisores anónimos que ayudaron a mejorar sustancialmente el escrito.

\section{RESUMEN}

Introducción: Los estudios dendrocronológicos en México se han basado principalmente en las coníferas, mientras que las especies de madera dura han sido poco estudiadas. Este ha sido el caso del género Quercus, con una alta diversidad taxonómica en el país pero que no ha sido estudiado con fines dendrocronológicos, a pesar de los valores ecológicos y económicos de sus especies.

Objetivo: En la presente investigación se determinó el potencial dendroclimático de Quercus sideroxyla en el noroeste de México, y su relación con variables climáticas como precipitación y temperatura.

Métodos: La investigación se desarrolló en el estado de Durango en el ejido Chavarría Viejo en las coordenadas $\left(23^{\circ} 43^{\prime} \mathrm{N} \& 105^{\circ} 33^{\prime} \mathrm{W}\right)$, se recolectaron muestras de 5 a $7 \mathrm{~cm}$ en dos sitios bajo aprovechamiento forestal y se procesaron mediante técnicas dendrocronológicas convencionales.

Resultados: Se desarrolló una cronología de ancho de anillo total, la cual se compuso por 30 muestras de 16 árboles para el período de 1917 a 2018 (101 años). Se obtuvieron valores de intercorrelación entre series de 0.43 , de sensibilidad media de 0.36 , relación señal-ruido de 3.53 y autocorrelación de primer orden (0.58). En cuanto a la relación clima-crecimiento, los valores de índice de ancho de anillo se correlacionaron con datos de la estación climática más cercana al sitio de estudio; donde la precipitación invierno-primavera (enero-mayo) fue la variable de mayor influencia en el crecimiento de la especie.

Conclusiones: Con base en los parámetros dendrocronológicos se demuestra el alto potencial de la especie para ser empleada en estudios dendroclimáticos en la región, la respuesta de la especie a la precipitación es similar al de las coníferas con las que cohabita.

Palabras clave: anillos de crecimiento; características anatómicas; dendrocronología; influencia climática; roble.

\section{REFERENCIAS}

Acosta-Hernández, A., Pompa-García, M., \& Camarero, J. (2017). An updated review of dendrochronological investigations in Mexico, a megadiverse country with a high potential for tree-ring sciences. Forests, 8(5), 173-184. https://doi.org/10.3390/f8050160

Bartens, J., Grissino-Mayer, H. D., Day, S. D., \& Wiseman, P. E. (2012). Evaluating the potential for dendrochronological analysis of live oak (Quercus virginiana
Mill.) from the urban and rural environment, an exploratory study. Dendrochronología, 30(1), 15-21. https://doi.org/10.1016/j.dendro.2011.04.002

Cerano Paredes, J., Villanueva Díaz, J., Valdez Cepeda, R. D., Constante García, V., Luis González Barrios, J., \& Estrada Ávalos, J. (2012). Precipitación reconstruida para la parte alta de la cuenca del río Nazas, Durango. Revista Mexicana de Ciencias Forestales, 3(10), 7-23.

Chávez-Gándara, M. P., Cerano-Paredes, J., Nájera-Luna, J. A., Pereda-Breceda, V., Esquivel-Arriaga, G., Cervantes-Martínez, R., \& Corral-Rivas, S. (2017). Reconstrucción de la precipitación invierno-primavera con base en anillos de crecimiento de árboles para la región de San Dimas, Durango, México. BOSQUE, 38(2), 387-399. https://doi.org/10.4067/ S0717-92002017000200016

Constante-García, V. (2015). Aplicación de técnicas dendrocronológicas en Pinus pseudostrobus Lindl. Y Quercus canbyi Trel. para analizar la influencia climática y el cambio de uso de suelo. Universidad Autónoma de Nuevo León.

Constante-García, V., Villanueva-Díaz, J., Cerano-Paredes, J., \& Estrada-Ávalos, J. (2009). Medición y calidad de fechado en anillos de árboles. Instituto Nacional de Investigaciones Forestales.

Cook, E. R., \& Holmes, R. L. (1986). User manual for ARSTAN Laboratory of Tree Ring Research. University of Arizona.

Cruz-Cobos, F., De Los Santos-Posadas, H. M., \& Valdez-Lazalde, J. R. (2008). Sistema compatible de ahusamiento-volumen para Pinus cooperi Blanco en Durango, México. Agrociencia, 42, 473-485.

De La Paz Pérez-Olvera, C., \& Dávalos-Sotelo, R. (2008). Algunas características anatómicas y tecnológicas de la madera de 24 especies de Quercus (encinos) de México. Madera y Bosques, 14(3), 43-80.

Delgado, C. S. (2000). Aplicaciones estadísticas en análisis dendrocronológicos. En F. A. Roig (Ed.), Dendrocronología en América Latina (pp. 79-102). Editorial de la Universidad Nacional de Cuyo.

Díaz-Rámirez, B., Villanueva-Díaz, J., \& Cerano-Paredes, J. (2016). Reconstrucción de la precipitación estacional con anillos de crecimiento para la región hidrológica Presidio-San Pedro. Madera y Bosques, 22(1), 111-123.

Fritts, H.C. (1976). Tree rings and climate. Academic Press.

García, E. (2004). Modificaciones al sistema de clasificación climática de Köppen. Universidad Nacional Autónoma de México. 
Grissino-Mayer, H. D. (2001). Evaluating Crossdating Accuracy: A Manual and Tutorial for the Computer Program COFECHA. Tree-Ring Research, 57(2), 205-221.

Herrera-Fernández, A. C., Carrillo-Parra, A., PedrazaBucio, F. E., Correa-Méndez, F., Herrera-Bucio, R., López-Albarrán, P., \& Rutiaga-Quiñones, J. G. (2017). Densidad, composición química y poder calorífico de la madera de tres especies de encino (Quercus candicans, Q. laurina y Q. rugosa). Ciencia Nicolaita, 72, 136-154.

Himmelsbach, W. (2009). Caracterización de bosques mixtos de pino-encino en la Sierra Madre Oriental en México considerandoel factor limitante hídrico. Universidad Autónoma de Nuevo León.

Holmes, R. L. (1983). Computer-assisted quality control in tree-ring dating and measurement. Tree-Ring Bulletin, 43, 69-78.

Luna-José, A. D. L., Montalvo-Espinosa, L., \& RendónAguilar, B. (2003). Los usos no leñosos de los encinos en México. Boletín de la Sociedad Botánica de México, 72, 107-117.

Ortiz-Quijano, A. B., Cuevas-Cardona, C., VillanuevaDíaz, J., López-Mata, L., \& Sánchez-González, A. (2018). Dendrochronological Reconstruction of Environmental History of Fagus grandifolia Subsp. Mexicana in Mexico. Tree-Ring Research, 74(1), 108-119. https://doi.org/10.3959/1536-1098-74.1.108

Pompa-García, M., Dávalos-Sotelo, R., Rodríguez-Téllez, E., \& Aguirre-Calderón, O. A. (2014). Sensibilidad climática de tres versiones dendrocronológicas para una conifera mexicana. Madera y Bosques, 20(3), $139-151$.

Romero Rangel, S., Rojas Zenteno, E. C., \& Aguilar Enríquez, M. L. (2002). El género Quercus (Fagaceae) en el Estado de México. Annals of the Missouri Botanical Garden, 89, 551-593.

Servicio Meteorológico Nacional-Comisión Nacional del Agua. (2019). Base de datos climatológicos. https:// smn.cna.gob.mx/climatologia/Diarios

Speer, J. (2010). Fundamentals of tree-ring research. University of Arizona Press.

Stahle, D. W. (1999). Useful strategies for the development of tropical tree-ring chronologies. IAWA Journal, 20(3), 249-253. https://doi. org/10.1163/22941932-90000688
Stokes, M. A., \& Smiley, T. L. (1968). An Introduction to Tree-Ring Dating. University of Chicago Press.

Swetnam, T. W., Thompson, M. A., \& Sutherland, E. K. (1985). Using dendrochronology to measure radial growth of defoliated trees. US Department of Agriculture, Forest Service, Cooperative State Research Service.

Valenzuela Nuñez, L. M., \& Granados Sánchez, D. (2009). Caracterización fisonómica y ordenación de la vegetación en el área de influencia de El Salto, Durango, México. Revista Chapingo Serie Ciencias Forestales $y$ del Ambiente, 15(1), 29-42.

Villanueva-Díaz, J., Cerano-Paredes, J., Constante-García, V., Montez-González, L. E., \& Vázquez Selem, L. (2009a). Muestreo Dendrocronológico: colecta, prepración y procesamiento de núcleos de crecimiento y secciones transversales. Gómez Palacio.

Villanueva-Díaz, J., Cerano-Paredes, J., Rosales-Mata, S., Arrocena-López, J. C., Stahle, D. W., Ruiz-Corral, J. A., \& Martinez-Sifuentes, A. R. (2014). Variabilidad hidroclimática reconstruida con anillos de árboles para la cuenca alta del Río Mezquital, Durango. Revista Mexicana de Ciencias Agrícolas, 5(10), 1897-1912.

Villanueva-Díaz, J., Cerano-Paredes, J., Stahle, D. K., Constante-García, V., \& Estrada, J. (2010). Estandarización y desarrollo de series dendrocronológicas en México (Vol. 16). Gómez Palacio, Durango.

Villanueva-Díaz, J., Cerano-Paredes, J., Stahle, D. W., Therrell, M. D., Cleaveland, M. K., \& Sánchez-Cohen, I. (2004). Elementos básicos de la dendrocronología y sus aplicaciones en México. Gómez Palacio.

Villanueva Díaz, J., Fulé, P. Z., Cerano-Paredes, J., Estrada Ávalos, J., \& Sánchez Cohen, I. (2009b). Reconstrucción de la precipitación estacional para el barlovento de la Sierra Madre Occidental con anillos de crecimiento de Pseudotsuga menziesii (Mirb) Franco. Revista Ciencia Forestal en México, 34(105), 39-71.

VoorTech. (2014). Measure J2X (V5.0x). [Software] VoorTech. http://www.voortech.com/projectj2x/

Wigley, T. M. L., Briffa, K. R., \& Jones, P. D. (1984). On the Average Value of correlated Time Series, with Applications in Dendroclimatology and Hidrometeorology. Journal of Climate and Applied Meteorology, 23(2), 201-213. 